\title{
TLR4 May Accelerate Hypoxia Reaction to Promote the Occurrence and Progress of Cervical Lesions by Infected Pathogenic Microorganisms Other than HPV*
}

\author{
Yanxiang Cheng ${ }^{1}$, Gantao Chen ${ }^{2}$, Xiaohang Wang ${ }^{1}$, Yuhui Huang ${ }^{1}$, Jinli Ding ${ }^{1}$, Jinling Huang ${ }^{1}$, \\ Li Hong ${ }^{1 \#}$
}

${ }^{1}$ Renmin Hospital of Wuhan University, Wuhan, China; ${ }^{2}$ Renmin Hospital of Xiantao City of Hubei Province, Xiantao, China.

Email: " chengmin.jjjj@yahoo.com.cn

Received February $6^{\text {th }}$, 2013; revised March $10^{\text {th }}, 2013$; accepted March $18^{\text {th }}, 2013$

Copyright (c) 2013 Yanxiang Cheng et al. This is an open access article distributed under the Creative Commons Attribution License, which permits unrestricted use, distribution, and reproduction in any medium, provided the original work is properly cited.

\begin{abstract}
Objective: To explore what role the inflammatory immune response is playing in the occurrence and development of HPV related cervical disease. Methods: To detect the expression of TLR4, HIF-1 $\alpha$ and HPV16 E7 in the specimens of HPV related cervical lesions, for example normal cervix, cervicitis, cervical intraepithelial neoplasia and cervical cancer by RT PCR methods. Results: The expression of TLR4 and HIF- $1 \alpha$ ascent from normal cervix to cervicitis, then to CIN tissue, and then to cervical cancer $(\mathrm{P}<0.05)$. The expression of HPV16 E7 was higher in cervicitis than that in normal cervix $(\mathrm{P}<0.05)$ while there was no significant ascendance from cervicitis to CIN tissue, either from CIN tissue to cervical cancer although it seemed ascending $(\mathrm{P}>0.05)$. Conclusion: TLR4 may promote the occurrence and progress of cervical lesions by accelerating the hypoxia reaction. And the natural immune reaction mediated by TLR4 may be correlated to other pathogenic microorganisms infecting the cervical tissue, rather than HPV.
\end{abstract}

Keywords: TLR4; HIF-1 $\alpha$; Cervical Lesions; HPV

\section{Introduction}

Toll-like receptor 4 (TLR4), the natural receptor of lipopolysaccharide, plays an important role in the regulation of acute inflammatory response, cellular signal transduction and apoptosis, and TLR4 is a natural immune regulatory factor. The expression of TLR4 can be detected in a variety of inflammatory diseases [1,2], suggesting that TLR4-mediated innate immune response is the starting point of the inflammatory diseases. TLR4 not only identifies exogenous pathogens, but also identifies endogenous substances and their degradation products [3], which can activate the MAPK pathway, the signal of NF- $\kappa$ B and hypoxia-inducible factor- $1 \alpha$ (HIF- $1 \alpha$ ) [4]. And HIF- $1 \alpha$ is closely related with the occurrence and development of cervical cancer. In this study, by researching the expression of TLR4 in HPV infection related cervical diseases, we researched the relationship between HPV infection-related diseases and the expression of

\footnotetext{
*This research was sponsored by China's Post-Doctoral Science Fund (numbered 2011M500857) and Natural Science Fund of Hubei Province (numbered ZRY139).

${ }^{\#}$ Corresponding author.
}

TLR4, and preliminarily explored the interaction between HIF- $1 \alpha$ and TLR4.

Toll-like receptors (TLRs) are the "Sentinel" that perceive exogenous microbial stimulation, and widely exist in the natural immune cell membrane, binding their ligands, playing a decisive role in the innate and adaptive immune response caused by microbial infection or tissue injury. The majority of TLRs express in the surface of antigen presenting cells in normal circumstances, for example, in the surface of phagocytic cells (including macrophages, neutrophils, dendritic cells). TLRs family has at least 13 members, among which TLR4 mainly recognizes invading bacteria [5].

\section{Materials and Methods}

\subsection{Specimens}

The study objects were recruited from the TCT (Thin Prep Cytologic Test) suspicious patients in the Gynecological clinic and census, who underwent colposcopy and cervical multipoint biopsy. According to the results, patients were diagnosed as cervicitis complicating HPV 
infection, CIN (cervical intraepithelial neoplasia) or cervical cancer. Cervical exfoliated cell specimens were taken for fluorescent quantitative PCR to identify highrisk HPV16 infection patients, who were included in the observed objects. All patients were new cases, and had no history of hormone therapy, radiotherapy, chemotherapy or cervical topical treatment. All patients were divided into HPV-infected cervicitis group, CIN group and cervical cancer group. About 20 cases were included in each subgroup, 20 cases of healthy cervical tissue from the patients who had hysterectomy due to uterine fibroids or uterine bleeding, without HPV infection or cervical lesions were used as control. The age of the patients included were from 16 years old to 64 years old, with the mean age of $32.7 \pm 6.3$ years old, and there was no significant difference with age in each group. All human tissue samples were used with ethics committee approval and consent from the patients in accordance with the guidelines of the Ethical Committee of the Renmin hospital of Wuhan University Medical Center. The specimen was diagnosed by two senior pathologists in consensus.

\subsection{Detection of HPV16 Infection}

Extraction of the DNA from cervical cells: We collected $0.5 \mathrm{ml}$ from each sample and centrifuged for $15 \mathrm{~min}$ by $13,000 \mathrm{~g}$, and discarded the supernatant. DNA was extracted with QIAMin kit (QIAGEN), then underwent PCR amplification and flow-through hybridization on the lowdensity gene chips, which included 21 hybridization probes, such as $6,11,42,43,44,16,18,31,33,35,39,45,51$, 52, 53, 56, 58, 59, 66, 68, CP8304. The samples were then made enzyme labeled colored. Those positive were presented blue-purple dots. We determined visual study afterwards, and judged the HPV subtypes of corresponding gene chips.

Detection of the target gene by RT-PCR: The fresh tissue was stored in liquid nitrogen, and was grounded into powder before experiment. Homogenate triturated tissue $0.5 \mathrm{~g}$ of each sample was lysed to extract total RNA, the A260/A280 purity was between 1.8 to 2.2 . Then the extracted total RNA underwent reverse transcript and PCR amplification. The product was subjected to gel electrophoresis, and then analyzed by gel imaging system. The mRNA of internal control $\beta$-actin was respectively amplified in the same system with TLR4, HIF- $1 \alpha$ and HPV16 E7 to reduce the impact of interference factors on experimental results, so after the density of all mRNA was analyzed by gel imaging system, we took the density of $\beta$-actin as an internal control, and the ratio of the density of the target gene and the endogenous control as the relative expression value of the target gene. Each experiment was repeated three times and averaged. All primers were provided in Table 1.

\subsection{Statistical Methods}

Data were expressed as means \pm S.E.M. Differences among groups were tested by one-way analysis of variance and linear correlation analysis. A value of $\mathrm{P}<0.05$ was considered to be significantly different.

\section{Results}

\subsection{The Expression of TLR4 mRNA in Cervical Tissues}

We researched the relationship between TLR4's expression and HPV infection related diseases by detecting the content of TLR4 RNA. RT-PCR results suggested that the expression of TLR4 gradually increased from the normal cervical tissues, HPV16 infected cervicitis tissues, CIN tissues to the cervical cancer tissues (Figures 1 (a) and (d)). After the gray value analysis, the relative expression values of TLR4 of each group were shown in Table 1, the difference was extremely significant. The results suggested that there was a certain correlation with TLR4's expression and the cervix and the development of cervical cancer.

\subsection{The Expression of HIF-1 $\alpha$ mRNA in Cervical Tissues}

Both HIF- $1 \alpha$ and TLR4 are associated with cervical cancer, we detected the changes of HIF- $1 \alpha$ RNA's content in each group to explore their relationships. The RNA content of HIF- $1 \alpha$ of each group was similar with TLR4, after the gray value analysis, HIF- $1 \alpha$ expression value of each group were shown in Table 1, the difference was extremely significant by statistical analysis (Figures 1 (b) and (d)). The results suggested that the expression of HIF$1 \alpha$ in cervical was positively correlated with the expression of TLR4.

\subsection{The Change of HPV16 E7 RNA Content}

We explored the relevance of HPV16 infection and cer-

Table 1. The primer sequence of every target gene.

\begin{tabular}{ccc}
\hline Target gene & \multicolumn{2}{c}{ Primer sequence } \\
\hline \multirow{2}{*}{ TLR4 } & Apstream & downstream \\
& CACTTGGACCTTTCC & GGGTTCAGGGAC- \\
& AGGTCTAAAGA \\
HIF-1 $\alpha$ & TCAAAGTCGGA- & CCCTGCAG- \\
& CAGCCTCA & TAGGTTTCTGCT \\
HPV16 E7 & ATGAGCAAT- & AGCCCAT- TAA- \\
& TACCCGACAGC & CAGCTCTTGC \\
$\beta$-actin & AAGAGAGG- & TACATGGCTGG- \\
& CATCCTCACCCT & GGTGTTGAA \\
\hline
\end{tabular}


TLR4 May Accelerate Hypoxia Reaction to Promote the Occurrence and Progress
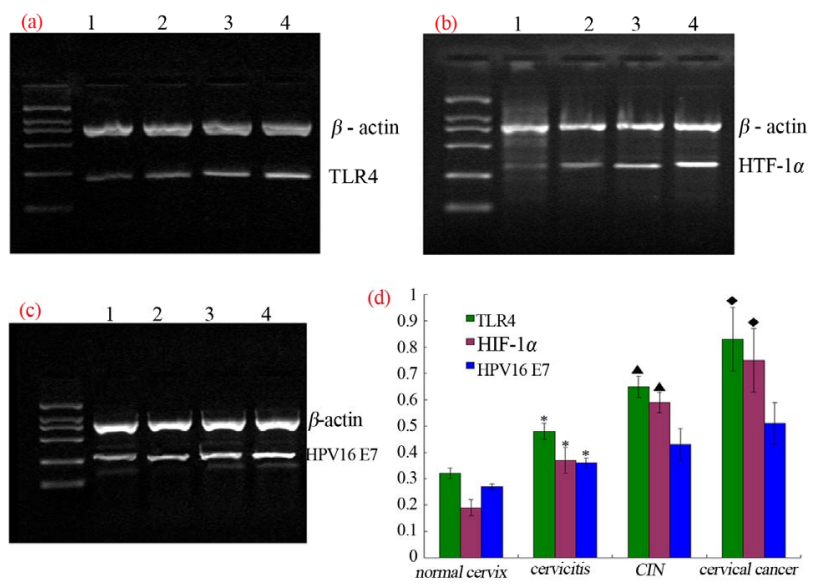

Figure 1. To detect the gene expression of TLR4, HIF-1 $\alpha$, HPV16 E7 (Figures 1 (a)-(c)) in different cervical tissue by RT-PCR gel electrophoresis image (lane 1: normal cervical; lane 2: HPV16 infected cervicitis; lane 3: CIN; lane 4: cervical cancer). (d) The expression of TLR4 and HIF-1 $\alpha$ was ascending from normal cervix to cervicitis, then to CIN tissue, and then to cervical cancer $(P<0.05)$. The expression of HPV16 E7 was higher in cervicitis than that in normal cervix $(P<0.05)$ while there was no significant ascendance from cervicitis to CIN tissue, either from CIN tissue to cervical cancer though it seemed ascending aparently $(P>0.05)$ (statistic difference as *, $\Delta, \diamond$ represent $\mathrm{P}<0.05$. *: normal cervix vs. cervicitis tissue, $\Delta$ : CIN tissue vs. cervicitis, $\diamond$ : CIN vs. cervical cancer).

vical lesions by detecting the changes of HPV16 E7 RNA's content in each group. The gray value analysis of HPV16 E7 RNA content showed that the expression of HPV16 E7 presented an upward trend from the normal cervix, to the cervicitis tissue and then to cervical cancer, but the difference was not statistically significant (Figures 1 (c) and (d)). The results showed that HPV16 induced the occurrence of cervical lesions, but the severity of cervical lesions was not necessarily associated with HPV loading doses.

\subsection{The Relevance Analysis of Expression Content of TLR4 and HIF-1 $\alpha$ in Cervical Cancer Tissues}

We made two-factor linear relevance analysis and hypothesis testing of correlation coefficient to TLR4 and HIF-1 $\alpha$ gene content in cervical cancer tissues, and the result showed a positive correlation $\mathrm{r}=0.491$, $\mathrm{P}<0.05$.

\subsection{The Relevance Analysis of Expression Content of TLR4 and HPV16 E7}

We made two-factor linear relevance analysis and hypothesis testing of correlation coefficient to TLR4 and HPV16 E7 gene content in cervical cancer tissues, and the result was $\mathrm{r}=0.121, \mathrm{P}=0.0612$.

\subsection{The Relevance Analysis of Expression Content of HIF-1 $\alpha$ and HPV16 E7}

We made two-factor linear relevance analysis and hypothesis testing of correlation coefficient to HIF-1 $\alpha$ and HPV16 E7 gene content in cervical cancer tissues, and the result was $r=0.207, P=0.0563$.

\section{Discussion}

Our study found that from the normal cervical tissues, HPV16 infected cervicitis tissues, CIN tissues to the cervical cancer tissues, the expression of TLR4 gradually increased and the difference was quite significant, the content of HIF-1 $\alpha$ RNA was similar to TLR4 and the difference was extremely significant, the expression of HPV16 E7 presented an upward trend but the difference had no significance. In cervical cancer tissues, the content of TLR4 and HIF-1 $\alpha$ 's gene was positively correlated and the difference was significant, the content of TLR4 and HPV16 E7's gene, HIF-1 $\alpha$ and HPV16 E7's gene showed no significant correlation.

Cervical cancer is the unique cancer whose cause is unclear in all human cancers so far. High-risk types of human papillomavirus are found in almost all cervical tissues of cervical cancer patients. Epidemiological investigation and laboratory research data have shown that human papillomavirus infection, especially high-risk virus HPV16 type, is the main epidemiological factor of cervical cancer [6,7]. If someone is infected with HPV, the virus gene E7 or E6 can be integrated into the epithetlial cells of the cervix, which may result in two outcomes: for people who have normal immune function, infection period is relatively short, they can usually get rid of the virus by their own immune system in about 8 - 10 months, which is called "transient infection"; however, if the virus is not cleared by the immune system, it can cause persistent infection, which then cause the hyperplasia and atypia changes of cervical cells, and eventually result in cancerization [8]. Therefore, it is reasonable to propose that the main reason of HPV16 infected cervical disease is the local immunomodulatory imbalance.

The pathogenesis of cervical cancer includes the inflammatory response process, which is mediated by the innate immune defense TLRs pathway. On one hand, the innate immune defense system can identify and clear infected HPV virus as much as possible, on the other hand, inflammatory chemotactic factors involved in the reaction can promote cervical epithelial dysplasia, andeven cancerization [9]. Previous study have shown that TLR4, which expresses in the surface of skin and mucous membranes, activates the innate immune response, regulates the release of proinflammatory cytokines and plays an important role in the immune response of skin and mu- 
cous membranes by identifying pathogenic microorganisms [10]. Meanwhile, TLR4-mediated inflamematory response is a common pathogenesis of many important diseases.

The activation of mitogen activated protein kinase (P44/ 42MAPK) pathway was regulated by proliferation and inflammatory signal [11,12]; in monocytes and macrophages, bacterial lipopolysaccharide (LPS) induced the activation of P44/42, activates NF- $\kappa \mathrm{B}$, regulated the inflammatory signal pathway, including the HIF- $1 \alpha$ signal pathway, which was involved in the occurrence and development of cervical cancer through pattern recognition receptor(PRR) [13]. The pattern recognition receptor Toll-like receptor 4 (TLR4) which recognizes bacterial's LPS is high-expressed in many diseases, TLR4-regulated signal passage can not only stimulate the body's innate immunity [14], but also plays a role in the occurrence and development of subsequent secondary immune response.

Our study found that the expression of TLR4 increased with the severity of lesions increasing in HPV infected cervical lesions, which showed that the expression of TLR4 in cervix was associated with the occurrence and development of cervical cancer. Although the expression of HPV16 E7 had a trend to increase in cervical lesions along with the severity of disease increases, but the difference was not significant, which showed that HPV16 induced the occurrence of cervical lesions, but the severity of cervical lesions was not necessarily associated with HPV loading doses (of course, the experiment detected HPV16 only, but hadn't fully detected HPV loading doses, which might cause results to a certain bias). The correlation analysis results showed that the expression of TLR4 in cervical cancer was not associated with HPV16 E7 $(r=0.121, P=0.0612)$, which suggested that the TLR4 signal passage might not be directly involved in the clearance process of HPV, but the cytokines from other pathogens infection mediated by it might indirectly mediate the process of the persistent infection and carcinogenesis of HPV; which meant that other pathogens infection cooperated with HPV pathogenic action by immunomodulatory reaction that occurred via TLR4 pathway. The study also showed that HIF- $1 \alpha$ was closely related to the development of cervical cancer, and its high expression in cervical cancer had a strong positive correlation with the expression of TLR4 ( $r=0.491, \mathrm{P}<0.05$ ), but no correlation with the expression of HPV16 E7, which suggested that the role of HIF-1 $\alpha$ in the occurrence and development of cervical cancer was associated with TLR4mediated immune response.

Therefore, according to our research, TLR4 is highly expressed in HPV-associated cervical lesions, the expression is correlated with HIF- $1 \alpha$, while has no correlation with HPV16. It suggests that there may be other pa- thogens such as bacteria induce TLR4 pathway im- munomodulatory reaction, which indirectly promote the process of HPV16 triggered cervical lesions. HIF- $1 \alpha$ is associated with TLR4's role in the development of cervical cancer. it means that HPV16 is the primary cause inducing the development of cervical lesions, but other pathogens may involve in the incidence of cervical cancerwhich activate TLR4 signal passage, and trigger the inflammatory response signal system, leads to the disorder of local immune environment, and finally aggravates the process of cervical lesions.

\section{REFERENCES}

[1] M. F. Abdul-Careem, M. Firoz Mian, A. E. Gillgrass, et al., "FimH, a TLR4 Ligand, Induces Innate Antiviral Responses in the Lung Leading to Protection against Lethal Influenza Infection in Mice,” Antiviral Research, Vol. 92, No. 2, 2011, pp. 346-355.

doi:10.1016/j.antiviral.2011.09.004

[2] G. Raicevic, M. Najar, K. Pieters, et al., "Inflammation and TLR Ligation Differentially Affect the Osteogenic Potential of Human Mesenchymal Stromal Cells (MSC) Depending on Their Tissue Origin,” Tissue Engineering Part A, Vol. 25, 2012.

[3] S. Challa, M. Woelfel, M. Guildford, et al., "Viral Cell Death Inhibitor MC159 Enhances Innate Immunity against Vaccinia Virus Infection,” Journal of Virology, Vol. 84, No. 20, 2010, pp. 10467-10476. doi:10.1128/JVI.00983-10

[4] M. Ramanathan, W. Luo, B. Csóka, et al., "Differential Regulation of HIF-1Alpha Isoforms in Murine Macrophages by TLR4 and Adenosine $\mathrm{A}_{2 \mathrm{~A}}$ Receptor Agonists," Journal of Leukocyte Biology, Vol. 86, No. 3, 2009, pp. 681-689. doi:10.1189/jlb.0109021

[5] J. Hu, D. Lou, B. Carow, et al., "LPS Regulates SOCS2 Transcription in a Type I Interferon Dependent AutocrineParacrine Loop,” PLoS One, Vol. 7, No. 1, 2012, Article ID: e30166. doi:10.1371/journal.pone.0030166

[6] C. B. Lu, M. J. Yang, L. Luo, et al., "Detection and Typing of Human Papillomavirus by a GeXP Based Multiplex PCR Assay,” Chinese Journal of Experimental and Clinical Virology, Vol. 25, No. 1, 2011, pp. 69-72.

[7] E. F. Dunne, M. Sternberg, L. E. Markowitz, et al., "Human Papillomavirus (HPV) 6, 11, 16, and 18 Prevalence among Females in the United States-National Health and Nutrition Examination Survey, 2003-2006: Opportunity to Measure HPV Vaccine Impact?" The Journal of Infectious Diseases, Vol. 204, No. 4, 2011, pp. 562-565.

[8] B. S. Bowser, S. Alam and C. Meyers, "Treatment of a Human Papillomavirus Type 31b-Positive Cell Line with Benzo[a]Pyrene Increases Viral Titer through Activation of the Erk1/2 Signaling Pathway,” Journal of Virology, Vol. 85, No. 10, 2011, pp. 4982-4992. doi:10.1128/JVI.00133-11

[9] X. W. Lu, C. Y. Xiao and H. Ye, "The Research Progress on the Correlationship of Cyto Factor and Human Pappi- 
loma Virus to Cervical Cancer," The Clinical Physician Magazine: Electronic Edition, Vol. 5, No. 14, 2011, pp. 4163-4166.

[10] A. M. Chaussé, O. Grépinet, E. Bottreau, et al., "Expression of Toll-Like Receptor 4 and Downstream Effectors in Selected Cecal Cell Subpopulations of Chicks Resistant or Susceptible to Salmonella Carrier State," Infection and Immunity, Vol. 79, No. 8, 2011, pp. 3445-3454. doi:10.1128/IAI.00025-11

[11] M. Bosmann, V. R. Patel, N. F. Russkamp, et al., "MyD88Dependent Production of IL-17F Is Modulated by the Anaphylatoxin C5a via the Akt Signaling Pathway," The FASEB Journal, Vol. 25, No. 12, 2011, pp. 4222-4232. doi:10.1096/fj.11-191205
[12] M. G. De Boer, H. Jolink, C. J. Halkes, et al., "Influence of Polymorphisms in Innate Immunity Genes on Susceptibility to Invasive Aspergillosis after Stem Cell Transplantation,” PLoS One, Vol. 6, No. 4, 2011, Article ID: e18403.

[13] M. Nakamura, J. M. Bodily, M. Beglin, et al., "HypoxiaSpecific Stabilization of HIF-1Alpha by Human Papillomaviruses," Virology, Vol. 387, No. 2, 2009, pp. 442-448. doi:10.1016/j.virol.2009.02.036

[14] G. Kleveta, K. Borzęcka, M. Zdioruk, et al., "LPS Induces Phosphorylation of Actin-Regulatory Proteins Leading to Actin Reassembly and Macrophage Motility," Journal of Cellular Biochemistry, Vol. 113, No. 1, 2012, pp. 80-92. doi:10.1002/jcb.23330 